\title{
EFFECT OF SPRAYING OF PROSOPIS FARCTA EXTRACT, FOLICIST AND ABO NAJEMH20 ON GROWTH OF POMEGRANATE TRANSPLANT VAR. WONDERFUL CALIFORNIA
}

\author{
HASAN SALEEM NABI \\ Dept. of Horticulture, College of Agricultural Engineering Sciences, University of Duhok, \\ Kurdistan Region - Iraq
}

(Received: September 1, 2021; Accepted for Publication: October 7, 2021 )

\begin{abstract}
This investigation was carried out during growing season (2019-2020) in the lathhouse of College of Agricultural Engineering Sciences, University of Duhok, Kurdistan region, Iraq. To investigate the effect of spraying with Prosopis farcta $(0,100$ and 200 ppm), Folicist $(0,50$, and 100 ppm) and Abo-najemh20 (50, 75 and $150 \mathrm{ppm}$ ) on growth of Pomegranate transplant. The results show that the all studied parameters were increased by increasing the concentration of Prosopis farcta especially at (200 ppm) and Folicist at (100ppm) and Abo-najemh20 at (150ppm). The best interaction between Prosopis farcta and Folicist was (200ppm and $100 \mathrm{ppm})$ respectively. Also, the interaction of Prosopis farcta at 200ppm with Abo-najemh20 at 150ppm lead to enhancing most studied characteristics. And the interaction between Folicist at 100ppm $+150 \mathrm{ppm}$ had a significant difference in improving the studied parameters. However, in triple combinations it found that the Prosopis farcta at 200ppm + Folicist at 100ppm + Abo najemh20 at $150 \mathrm{ppm}$ was superior interaction compared with all other combination treatment including control treatment.
\end{abstract}

KEY WORDS: Pomegranate, Prosopis farcta, Folicist, Abo najemh20 and organic acid

\section{INTRODUCTION}

$\mathbf{P}$ Oomegranate (Punica granatum L.), belonging to the family Punicaceae, is one of the favorite table fruits in the world, for its refreshing juice with nutritional value and medicinal purpose (Gosh et.al., 2009). Cultivation of Pomegranate now a day is considered a highly profitable and productive agriculture business in the world. It is widely cultivated throughout India, Iran, China, Turkey, USA, Spain, Azerbaijan, Armenia, Afghanistan, Uzbekistan, the Middle East, Pakistan, Tunisia and Israel, dry regions of Southeast Asia, Peninsular Malaysia, the East Indies and tropical Africa. The leading countries in the world for production of pomegranate were India (Sharma et.al., 2014). In Kurdistan region of Iraq, the pomegranate commercially had a significant value, even there were a wide range of preferred alternative fruits because of it uses in making shaperman (ketchup) and eating dried seeds of pomegranate fruits. Prosopis Lagonychium farctum genus is an underutilized legume plant that belongs to the Leguminosae family Its importance is a small fodder, but generally viewed as not more than an invasive herbs. (Pasiecznik et.al., 2004). Prosopis genus comprises 44 species, including P. juliflora, P. farcta, P. velutina, P. glandulosa, P. laevigata, P. pallida, P. cineraria, etc. The range of Prosopis farcta is similar to that of Prosopis cineraria from India to Iran, but then extends to the west and north. It is widespread in the Middle East, and is also found in Cyprus, Turkey, and Ukraine and along the North African coast as far as Algeria. No specific habitat preferences were observed, with a wide climatic range. (Pasiecznik et.al., 2004). Prosopis is mainly distributed in arid, semi-arid, tropical, and subtropical countries, such as America, India, Argentina, Chile, Kenya, and Pakistan (PASIECZNIK, 2001). Prosopis faracta is a good source of phenolic compounds and flavonoids such as rutin, myricetin and caffeic acid derivatives (Harzallah-Skhiri \& BenJannet, 2005) Folicist is used in many agricultural crops in order to improve seedling growth are required to achieve optimal growth and yield (Ziosi et.al., 
2013). Folicist are reported to play a significant role in pomegranate (Chaudhari and Desai, 1993). Hence, an attempt has been made to find Folicist and their doses for improving fruit set and yield in pomegranate cv. Ruby, under West Bengal conditions (Chaudhari \& Desai, 1993). The most important components of organic matter have been reported in Abo-Najemh20. Which was a strong role in increasing microbial activity, it is considered as a bio-stimulant for plant growth; Promotes absorption of nutrients as a chelating agent and improves vegetative properties, nutritional status and leaf pigments (Eissa Fawzia et.al., 2007).

The aim of this study was to improve the quality of pomegranate seedling to be ready for buddling and grafting for next year this by using Prosopis farcta Extract, Folicist and Abo najemh20. Also, to determine the effect of Prosopis farcta for plant due to there were limited researchers on this plant.

\section{MATERIAL AND METHODS}

This study was carried out during growing season of 2019-2020 on Pomegranate (Punica granatum var Wonderful California) seedling in the lath house of Horticulture department. College of Agricultural Engineering Sciences. To improve the growth of seedlings by spraying application three concentrations of Prosopis farcta Extract with (0, 100 and $200 \mathrm{ppm})$, Folicist (0, 50 and $100 \mathrm{ppm})$ and Abo najemh20 $(0,75$ and $150 \mathrm{ppm})$ in May and June 2020. The ripen fruits of Prosopis farcta plants were collected from Peromara village. Duhok, in July 2018. The fruits were dried at $75 \pm 5{ }^{\circ} \mathrm{C}$ for two days. Samples were powdered by a mechanical grinder and were stored at a refrigerated temperature in a dark environment to avoid light exposure then dissolved in alcohol to make a stock solution. The data was collected and analyzed by using factorial RCBD and Duncan's test (Al-Rawi, \& Khalafalla, 2000). $(3 * 3 * 3)$ with three blocks to become (81) experimental units each of them consists of three seedlings. The data was analyzed by using (SAS, 2015) Programme.

\section{RESULTS AND DISCUSSIONS}

\subsection{Transplant height $(\mathrm{cm})$}

The data in table (1) showed that the Prosopis farcta had a significant effect on increasing the highest of pomegranate transplant as compared with control, the same effect was found with Folicist and abo-Najem h20. The height of pomegranate transplant in interactions between Prosopis farcta and Folicist got highest value with $200 \mathrm{ppm}$ Prosopis farcta and $100 \mathrm{ppm}$ Folicist which was $(143.22 \mathrm{~cm})$ as compared with other interactions, the superior interactions that affecting transplant height was between (200 ppm Prosopis farcta +150 ppm AboNajemh20) (100 ppm Folicist +150 ppm AboNajemh20) which were $(137.78 \mathrm{~cm})(137.89 \mathrm{~cm})$ respectively. The triple interaction between Prosopis farcta, Folicist and Abo-Najemh20 had a significant effect in enhancing the height of pomegranate transplants especially at $(200 \mathrm{ppm}$ Prosopis farcta, $100 \mathrm{ppm}$ Folicist and $150 \mathrm{ppm}$ Abo-Najemh20) which record $(150 \mathrm{~cm})$ compared with all other combinations treatments and control treatment which record $(94.67 \mathrm{~cm})$

Table (1): Effect of Prosopis farcta, Folicist and Abo najemh20 and their interactions on Hight $(\mathrm{cm})$ of Pomegranate transplant

\begin{tabular}{|c|c|c|c|c|c|c|}
\hline $\begin{array}{l}\text { Prosopis farcta } \\
\qquad(p p m)\end{array}$ & $\begin{array}{c}\text { Folicist } \\
\text { (ppm) }\end{array}$ & $\begin{array}{c}\text { Abo- } \\
\text { najemh20 } \\
0 \text { ppm }\end{array}$ & $\begin{array}{c}\text { Abo- } \\
\text { najemh20 } \\
75 \text { ppm }\end{array}$ & $\begin{array}{c}\text { Abo- } \\
\text { najemh20 } \\
150 \text { ppm }\end{array}$ & $\begin{array}{l}\text { Prosopis } \\
\text { farcta * } \\
\text { Folicist }\end{array}$ & Prosopis farcta \\
\hline \multirow[t]{3}{*}{0} & 0 & $94.67 \mathrm{~m}$ & $99.00 \mathrm{~lm}$ & $102.67 \mid$ & $98.78 \mathrm{~h}$ & $112.93 \mathrm{c}$ \\
\hline & 50 & $111.00 \mathrm{k}$ & $116.33 \mathrm{j}$ & $119.67 \mathrm{~h}-\mathrm{j}$ & $115.67 \mathrm{~g}$ & \\
\hline & 100 & $122.33 \mathrm{f}-\mathrm{i}$ & $123.00 \mathrm{f}-\mathrm{i}$ & $127.67 \mathrm{~d}-\mathrm{f}$ & $124.33 \mathrm{e}$ & \\
\hline \multirow[t]{3}{*}{100} & 0 & $117.67 \mathrm{ij}$ & $121.00 \mathrm{~g}-\mathrm{j}$ & $121.67 \mathrm{~g}-\mathrm{j}$ & $120.11 \mathrm{f}$ & $126.93 \mathrm{~b}$ \\
\hline & 50 & $124.67 d-h$ & $127.67 \mathrm{~d}-\mathrm{f}$ & 129.33 de & $127.22 \mathrm{~d}$ & \\
\hline & 100 & $129.67 \mathrm{~d}$ & $134.67 \mathrm{c}$ & $136.00 \mathrm{c}$ & $133.44 \mathrm{c}$ & \\
\hline \multirow[t]{3}{*}{200} & 0 & $119.67 \mathrm{~h}-\mathrm{j}$ & 124.00 e-h & $126.00 \mathrm{~d}-\mathrm{g}$ & $123.22 \mathrm{e}$ & $134.33 \mathrm{a}$ \\
\hline & 50 & $135.67 \mathrm{c}$ & $136.67 \mathrm{bc}$ & $137.33 \mathrm{bc}$ & $136.56 \mathrm{~b}$ & \\
\hline & 100 & $138.33 \mathrm{bc}$ & $141.33 \mathrm{~b}$ & $150.00 \mathrm{a}$ & $143.22 \mathrm{a}$ & \\
\hline \multicolumn{2}{|c|}{ Abo-najemh20 } & $121.52 \mathrm{c}$ & $124.85 b$ & $127.81 \mathrm{a}$ & & Folicist \\
\hline
\end{tabular}




\begin{tabular}{|c|c|c|c|c|c|c|}
\hline Prosopis farcta & 0 & $109.33 \mathrm{~h}$ & $112.78 \mathrm{~g}$ & $116.67 \mathrm{f}$ & & \\
\hline \multirow[t]{2}{*}{ * Abo-najemh20 } & 100 & $124.00 \mathrm{e}$ & $127.78 \mathrm{~d}$ & $129.00 \mathrm{~cd}$ & & \\
\hline & 200 & $131.22 \mathrm{c}$ & $134.00 \mathrm{~b}$ & $137.78 \mathrm{a}$ & & \\
\hline \multirow{3}{*}{$\begin{array}{c}\text { Folicist } \\
{ }^{*} \text { Abo-najemh20 }\end{array}$} & 0 & $110.67 \mathrm{~g}$ & $114.67 \mathrm{f}$ & $116.78 \mathrm{f}$ & 0 & $114.04 \mathrm{c}$ \\
\hline & 50 & $123.78 \mathrm{e}$ & $126.89 \mathrm{~d}$ & $128.78 \mathrm{~cd}$ & 50 & $126.48 \mathrm{~b}$ \\
\hline & 100 & $130.11 \mathrm{c}$ & $133.00 \mathrm{~b}$ & $137.89 \mathrm{a}$ & 100 & $133.67 \mathrm{a}$ \\
\hline
\end{tabular}

Means of each factor and their interactions followed with the same letters are not significantly different from each other's according to Duncans multiple range test at 5\% level.

\subsection{Stem diameter (mm)}

It clearly shown in table 2 that the three studied factors play a significant role in improving the dimeter of pomegranate transplant stems especially at high concentration as compared with untreated seedling. The stem diameter got highest value in dual interaction between $(200 \mathrm{ppm}$ Prosopis farcta $+100 \mathrm{ppm}$
Folicist) which recorded $(11.33 \mathrm{~mm})$. the third concentration of both dual interactions (Prosopis farcta + Abo-najemh20) (Folicist + Abonajemh20) as compared with control. The high value of stem diameter $(12 \mathrm{~mm})$ was acquired with 200 ppm Prosopis farcta, 100 ppm Folicist and $\quad 150 \quad$ ppm Abo-Najemh20

Table (2): Effect of Prosopis farcta, Folicist and Abo najemh20 and their interactions on Stem Diameter (mm) of Pomegranate transplant

\begin{tabular}{|c|c|c|c|c|c|c|}
\hline Prosopis farcta & Folicist & $\begin{array}{c}\text { Abo- } \\
\text { najemh20 } \\
0 \text { ppm }\end{array}$ & $\begin{array}{c}\text { Abo- } \\
\text { najemh20 } \\
75 \text { ppm }\end{array}$ & $\begin{array}{c}\text { Abo- } \\
\text { najemh20 } \\
150 \text { ppm }\end{array}$ & $\begin{array}{l}\text { Prosopis } \\
\text { farcta * } \\
\text { Folicist }\end{array}$ & Prosopis farcta \\
\hline \multirow[t]{3}{*}{0} & 0 & $6.17 \mathrm{j}$ & $7.00 \mathrm{i}$ & $7.83 \mathrm{~h}$ & $7.00 \mathrm{f}$ & $8.00 \mathrm{c}$ \\
\hline & 50 & $8.00 \mathrm{~h}$ & $8.17 \mathrm{gh}$ & $8.50 \mathrm{gh}$ & $8.22 \mathrm{e}$ & \\
\hline & 100 & $8.67 \mathrm{f}-\mathrm{h}$ & $8.67 \mathrm{f}-\mathrm{h}$ & $9.00 \mathrm{fg}$ & $8.78 \mathrm{~d}$ & \\
\hline \multirow[t]{3}{*}{100} & 0 & $8.17 \mathrm{gh}$ & $9.00 \mathrm{fg}$ & 9.50 ef & $8.89 \mathrm{~d}$ & $9.96 \mathrm{~b}$ \\
\hline & 50 & $10.00 \mathrm{de}$ & $10.00 \mathrm{de}$ & $10.33 c-e$ & $10.11 \mathrm{c}$ & \\
\hline & 100 & $10.67 \mathrm{~b}-\mathrm{d}$ & $11.00 \mathrm{bc}$ & $11.00 \mathrm{bc}$ & $10.89 a b$ & \\
\hline \multirow[t]{3}{*}{200} & 0 & $10.67 \mathrm{~b}-\mathrm{d}$ & $10.00 \mathrm{de}$ & $10.67 \mathrm{~b}-\mathrm{d}$ & $10.44 \mathrm{bc}$ & $10.78 \mathrm{a}$ \\
\hline & 50 & $10.33 \mathrm{c}-\mathrm{e}$ & $11.00 \mathrm{bc}$ & $10.33 c-e$ & $10.56 \mathrm{bc}$ & \\
\hline & 100 & $10.67 b-d$ & $11.33 \mathrm{ab}$ & $12.00 \mathrm{a}$ & $11.33 \mathrm{a}$ & \\
\hline \multicolumn{2}{|c|}{ Abo-najemh20 } & $9.26 \mathrm{c}$ & $9.57 b$ & $9.91 \mathrm{a}$ & & Folicist \\
\hline \multirow{3}{*}{$\begin{array}{l}\text { Prosopis farcta } \\
\text { * Abo-najemh20 }\end{array}$} & 0 & $7.61 \mathrm{f}$ & $7.94 \mathrm{f}$ & $8.44 \mathrm{e}$ & & \\
\hline & 100 & $9.61 \mathrm{~d}$ & $10.00 \mathrm{~cd}$ & $10.28 \mathrm{bc}$ & & \\
\hline & 200 & $10.56 \mathrm{ab}$ & $10.78 \mathrm{a}$ & $11.00 \mathrm{a}$ & & \\
\hline \multirow{3}{*}{$\begin{array}{c}\text { Folicist } \\
\text { *Abo-najemh20 }\end{array}$} & 0 & $8.33 \mathrm{e}$ & $8.67 \mathrm{e}$ & $9.33 \mathrm{~d}$ & 0 & $8.78 \mathrm{c}$ \\
\hline & 50 & $9.44 \mathrm{~d}$ & $9.72 \mathrm{~cd}$ & $9.72 \mathrm{~cd}$ & 50 & $9.63 \mathrm{~b}$ \\
\hline & 100 & $10.00 \mathrm{bc}$ & $10.33 a b$ & $10.67 \mathrm{a}$ & 100 & $10.33 a$ \\
\hline
\end{tabular}

Means of each factor and their interactions followed with the same letters are not significantly different from each other's according to Duncans multiple range test at 5\% level.

\subsection{Number of Branches per transplant}

The obtained data indicated a significant variation of Prosopis farcta in term of number of branches of pomegranate transplant. On contrary, the effect of Folicist was significant on number of branches with the supreme value measured for transplant supplied with (100 ppm) of Folicist which was (10.11) when compared with control and $(50 \mathrm{ppm})$ of
Folicist, the same effect was found in interaction between Folicist and Abonajemh20. Regarding the triple interaction between treatments, the supreme number of branches $(12.33 \mathrm{~cm})$ was measured for $(200 \mathrm{ppm}$ Prosopis farcta, 100 ppm Folicist and 150 ppm Abo-Najemh20). As shown in (Table 3). 
Table (3): Effect of Prosopis farcta, Folicist and Abo najemh20 and their interactions on Number of Branches of Pomegranate transplant

\begin{tabular}{|c|c|c|c|c|c|c|}
\hline Prosopis farcta & Folicist & $\begin{array}{c}\text { Abo- } \\
\text { najemh20 } \\
0 \text { ppm }\end{array}$ & $\begin{array}{c}\text { Abo- } \\
\text { najemh20 } \\
75 \text { ppm }\end{array}$ & $\begin{array}{c}\text { Abo- } \\
\text { najemh20 } \\
150 \text { ppm }\end{array}$ & $\begin{array}{l}\text { Prosopis farcta } \\
{ }^{*} \text { Folicist }\end{array}$ & Prosopis farcta \\
\hline \multirow[t]{3}{*}{0} & 0 & $3.33 \mathrm{k}$ & $5.00 \mathrm{i}-\mathrm{k}$ & $6.33 \mathrm{f}-\mathrm{j}$ & $4.89 \mathrm{~d}$ & $6.93 \mathrm{~b}$ \\
\hline & 50 & $5.00 \mathrm{i}-\mathrm{k}$ & $6.00 \mathrm{f}-\mathrm{k}$ & $7.33 \mathrm{e}-\mathrm{i}$ & $6.11 \mathrm{~cd}$ & \\
\hline & 100 & $8.33 \mathrm{~d}-\mathrm{g}$ & $9.67 \mathrm{~b}-\mathrm{e}$ & $11.33 \mathrm{a}-\mathrm{c}$ & $9.78 \mathrm{~b}$ & \\
\hline \multirow[t]{3}{*}{100} & 0 & $3.67 \mathrm{jk}$ & $5.33 \mathrm{~h}-\mathrm{k}$ & $5.33 \mathrm{~h}-\mathrm{k}$ & $4.78 \mathrm{~d}$ & $7.04 \mathrm{~b}$ \\
\hline & 50 & 7.33 e-i & 7.33 e-i & $7.67 \mathrm{e}-\mathrm{i}$ & $7.44 \mathrm{c}$ & \\
\hline & 100 & $6.33 \mathrm{f}-\mathrm{j}$ & $9.00 \mathrm{c}-\mathrm{e}$ & $11.33 \mathrm{a}-\mathrm{c}$ & $8.89 \mathrm{~b}$ & \\
\hline \multirow[t]{3}{*}{200} & 0 & $5.67 \mathrm{f}-\mathrm{j}$ & 7.67 e-i & 8.00 e-h & $7.11 \mathrm{c}$ & $9.22 \mathrm{a}$ \\
\hline & 50 & $7.67 \mathrm{e}-\mathrm{i}$ & $9.00 \mathrm{c}-\mathrm{e}$ & 10.00 a-e & $8.89 \mathrm{~b}$ & \\
\hline & 100 & 10.67 a-d & $12.00 \mathrm{ab}$ & $12.33 \mathrm{a}$ & $11.67 \mathrm{a}$ & \\
\hline \multicolumn{2}{|c|}{ Abo-najemh20 } & $6.44 \mathrm{c}$ & $7.89 \mathrm{~b}$ & $8.85 \mathrm{a}$ & \multicolumn{2}{|c|}{ Folicist } \\
\hline Prosopis farcta & 0 & $5.56 \mathrm{~d}$ & $6.89 \mathrm{~cd}$ & $8.33 \mathrm{bc}$ & & \\
\hline \multirow[t]{2}{*}{ * Abo-najemh20 } & 100 & $5.78 \mathrm{~d}$ & $7.22 \mathrm{c}$ & $8.11 \mathrm{c}$ & & \\
\hline & 200 & $8.00 \mathrm{c}$ & $9.56 \mathrm{ab}$ & $10.11 \mathrm{a}$ & & \\
\hline Folicist & 0 & $4.22 \mathrm{e}$ & $6.00 \mathrm{~d}$ & $6.56 \mathrm{~d}$ & 0 & $5.59 \mathrm{c}$ \\
\hline \multirow[t]{2}{*}{ *Abo-najemh20 } & 50 & $6.67 \mathrm{~d}$ & $7.44 \mathrm{~cd}$ & $8.33 \mathrm{c}$ & 50 & $7.48 \mathrm{~b}$ \\
\hline & 100 & $8.44 \mathrm{c}$ & $10.22 b$ & $11.67 \mathrm{a}$ & 100 & $10.11 \mathrm{a}$ \\
\hline
\end{tabular}

Means of each factor and their interactions followed with the same letters are not significantly different from each other's according to Duncans multiple range test at 5\% level.

\subsection{Number of leaves per one branch}

Results in table (4) revealed a significant difference of Prosopis farcta concentrations of (200ppm) in term of number of leaves per transplant. The same thing was true for the Folicist spraying influence and the leaf number was significantly differed from leaf number of control treatment. The Abo-najemh20 impact on number of leaves per transplant obtained a maximum average of number of leaves per transplant (134.70) in comparison with other treatments. Binary interaction of $200 \mathrm{ppm}$
Prosopis farcta +100 ppm Folicist lead to produce a maximum number of leaves. The interaction between Prosopis farcta and Abonajemh20 had a significant effect of increasing the number of leaves per transplant compared with control treatment. The same is true for the interaction between Folicist and Abo-najemh20. The interaction among (200 ppm Prosopis farcta +100 ppm Folicist +150 ppm Abo-Najemh20) creates a maximum average of number of leaves which counted (147.00) as compared with all other combination treatments.

Table (4): Effect of Prosopis farcta, Folicist and Abo najemh20 and their interactions on Number of Leaves of Pomegranate transplant

\begin{tabular}{|c|c|c|c|c|c|c|}
\hline Prosopis farcta & Folicist & $\begin{array}{c}\text { Abo- } \\
\text { najemh20 } \\
0 \text { ppm }\end{array}$ & $\begin{array}{c}\text { Abo- } \\
\text { najemh20 } \\
75 \text { ppm }\end{array}$ & $\begin{array}{c}\text { Abo- } \\
\text { najemh20 } \\
150 \text { ppm }\end{array}$ & $\begin{array}{c}\text { Prosopis farcta } \\
{ }^{*} \text { Folicist }\end{array}$ & Prosopis farcta \\
\hline \multirow[t]{3}{*}{0} & 0 & $118.33 \mathrm{~m}$ & $124.67 \mathrm{kl}$ & $125.33 \mathrm{i}-\mathrm{I}$ & $122.78 \mathrm{~h}$ & $125.48 \mathrm{c}$ \\
\hline & 50 & $125.00 \mathrm{j}-\mathrm{I}$ & $125.00 \mathrm{j}-1$ & $128.33 \mathrm{~g}-\mathrm{i}$ & $126.11 \mathrm{~g}$ & \\
\hline & 100 & $125.00 \mathrm{j}-\mathrm{I}$ & $127.67 \mathrm{~g}-\mathrm{k}$ & $130.00 \mathrm{f}-\mathrm{h}$ & $127.56 \mathrm{fg}$ & \\
\hline \multirow[t]{3}{*}{100} & 0 & 124.33 I & $128.00 \mathrm{~g}-\mathrm{j}$ & 132.67 ef & 128.33 ef & $129.44 \mathrm{~b}$ \\
\hline & 50 & $127.33 \mathrm{~h}-\mathrm{I}$ & $128.00 \mathrm{~g}-\mathrm{j}$ & $133.67 \mathrm{e}$ & $129.67 \mathrm{de}$ & \\
\hline & 100 & $127.33 \mathrm{~h}-\mathrm{I}$ & $130.67 \mathrm{e}-\mathrm{g}$ & 133.00 ef & $130.33 \mathrm{~d}$ & \\
\hline \multirow[t]{3}{*}{200} & 0 & $130.00 \mathrm{f}-\mathrm{h}$ & 133.00 ef & $137.33 \mathrm{~d}$ & $133.44 \mathrm{c}$ & $140.44 \mathrm{a}$ \\
\hline & 50 & $140.67 \mathrm{c}$ & $143.33 \mathrm{bc}$ & $145.00 a b$ & $143.00 \mathrm{~b}$ & \\
\hline & 100 & $142.00 \mathrm{c}$ & $145.67 \mathrm{ab}$ & $147.00 \mathrm{a}$ & $144.89 \mathrm{a}$ & \\
\hline \multicolumn{2}{|c|}{ Abo-najemh20 } & $128.89 \mathrm{c}$ & $131.78 \mathrm{~b}$ & $134.70 \mathrm{a}$ & \multirow{2}{*}{\multicolumn{2}{|c|}{ Folicist }} \\
\hline Prosopis farcta & 0 & $122.78 \mathrm{~h}$ & $125.78 \mathrm{~g}$ & 127.89 ef & & \\
\hline
\end{tabular}




\begin{tabular}{ccccccc}
\hline * Abo-najemh20 & 100 & $126.33 \mathrm{fg}$ & $128.89 \mathrm{e}$ & $133.11 \mathrm{~d}$ & & \\
& 200 & $137.56 \mathrm{c}$ & $140.67 \mathrm{~b}$ & $143.11 \mathrm{a}$ & & $128.19 \mathrm{c}$ \\
\hline Folicist & 0 & $124.22 \mathrm{e}$ & $128.56 \mathrm{~d}$ & $131.78 \mathrm{c}$ & 0 & $132.93 \mathrm{~b}$ \\
\cline { 2 - 6 }${ }^{*}$ Abo-najemh20 & 50 & $131.00 \mathrm{c}$ & $132.11 \mathrm{c}$ & $135.67 \mathrm{~b}$ & 50 & $134.26 \mathrm{a}$ \\
\cline { 2 - 6 } & 100 & $131.44 \mathrm{c}$ & $134.67 \mathrm{ab}$ & $136.67 \mathrm{a}$ & 100 & 0 \\
\hline
\end{tabular}

Means of each factor and their interactions followed with the same letters are not significantly different from each other's according to Duncans multiple range test at 5\% level.

\subsection{Total Chlorophyll (SPAD)}

The chlorophyll content in pomegranate transplant leaves had a significant difference with three studied factors especially at third concentration (200ppm, 100ppm and 150ppm) (Prosopis farcta, Folicist and Abo-Najemh20) respectively. The Chlorophyll content in leaves in interaction between Prosopis farcta and Folicist got highest value with 200 ppm Prosopis farcta and $100 \mathrm{ppm}$ Folicist which was (57.86 SPAD) as compared with other interactions, the superior interaction that affecting Chlorophyll was between $(200 \mathrm{ppm}$ Prosopis farcta +150 ppm Abo-Najemh20) (100 ppm Folicist +150 ppm Abo-Najemh20) which were (56.96 SPAD) (50.61 SPAD) respectively. The combined influence of the three factors on total chlorophyll percentage was profound and the record of the highest chlorophyll percentage in leaves (58.97 SPAD) belonged to (200 ppm Prosopis farcta + 100 ppm Folicist +150 ppm Abo-Najemh20) treatments as seen in table

Table (5): Effect of Prosopis farcta, Folicist and Abo najemh20 and their interactions on Chlorophyll content in leaves of Pomegranate transplant

\begin{tabular}{|c|c|c|c|c|c|c|}
\hline Prosopis farcta & Folicist & $\begin{array}{c}\text { Abo- } \\
\text { najemh20 } \\
0 \text { ppm }\end{array}$ & $\begin{array}{c}\text { Abo- } \\
\text { najemh20 } \\
75 \text { ppm }\end{array}$ & $\begin{array}{c}\text { Abo- } \\
\text { najemh20 } \\
150 \text { ppm }\end{array}$ & $\begin{array}{l}\text { Prosopis } \\
\text { farcta * } \\
\text { Folicist }\end{array}$ & Prosopis farcta \\
\hline \multirow[t]{3}{*}{0} & 0 & $34.73 r$ & $35.70 \mathrm{qr}$ & 39.37 op & $36.60 \mathrm{~h}$ & $39.17 \mathrm{c}$ \\
\hline & 50 & 39.83 op & 39.20 op & $37.73 \mathrm{pq}$ & $38.92 \mathrm{~g}$ & \\
\hline & 100 & $41.10 \mathrm{~m}-\mathrm{o}$ & $43.53 \mathrm{k}-\mathrm{m}$ & $41.33 \mathrm{~m}-\mathrm{o}$ & $41.99 \mathrm{f}$ & \\
\hline \multirow[t]{3}{*}{100} & 0 & 39.10 op & $42.53 \mathrm{l}-\mathrm{n}$ & $44.73 \mathrm{j}-1$ & $42.12 \mathrm{f}$ & $45.21 b$ \\
\hline & 50 & 40.83 no & $45.57 \mathrm{i}-\mathrm{k}$ & $46.40 \mathrm{~h}-\mathrm{j}$ & $44.27 \mathrm{e}$ & \\
\hline & 100 & $47.47 \mathrm{hi}$ & $48.73 \mathrm{gh}$ & 51.53 ef & $49.24 \mathrm{~d}$ & \\
\hline \multirow[t]{3}{*}{200} & 0 & $53.53 \mathrm{c}-\mathrm{e}$ & $55.33 \mathrm{~b}-\mathrm{d}$ & $55.93 \mathrm{bc}$ & $54.93 b$ & $55.36 \mathrm{a}$ \\
\hline & 50 & $50.57 \mathrm{fg}$ & $53.30 \mathrm{de}$ & $55.97 \mathrm{bc}$ & $53.28 \mathrm{c}$ & \\
\hline & 100 & $57.03 a b$ & $57.57 \mathrm{ab}$ & $58.97 \mathrm{a}$ & $57.86 \mathrm{a}$ & \\
\hline \multicolumn{2}{|c|}{ Abo-najemh20 } & $44.91 \mathrm{c}$ & $46.83 \mathrm{~b}$ & $48.00 \mathrm{a}$ & & icist \\
\hline Prosopis farcta & 0 & $38.56 \mathrm{~g}$ & $39.48 \mathrm{~g}$ & $39.48 \mathrm{~g}$ & & \\
\hline \multirow[t]{2}{*}{ * Abo-najemh20 } & 100 & $42.47 \mathrm{f}$ & $45.61 \mathrm{e}$ & $47.56 \mathrm{~d}$ & & \\
\hline & 200 & $53.71 \mathrm{c}$ & $55.40 \mathrm{~b}$ & $56.96 \mathrm{a}$ & & \\
\hline Folicist & 0 & $42.46 \mathrm{e}$ & $44.52 \mathrm{~d}$ & $46.68 \mathrm{c}$ & 0 & $44.55 \mathrm{c}$ \\
\hline \multirow[t]{2}{*}{ * Abo-najemh20 } & 50 & $43.74 \mathrm{de}$ & $46.02 \mathrm{c}$ & $46.70 \mathrm{c}$ & 50 & $45.49 \mathrm{~b}$ \\
\hline & 100 & $48.53 \mathrm{~b}$ & $49.94 \mathrm{a}$ & $50.61 \mathrm{a}$ & 100 & $49.70 \mathrm{a}$ \\
\hline
\end{tabular}

Means of each factor and their interactions followed with the same letters are not significantly different from each other's according to Duncans multiple range test at 5\% level.

\subsection{Number of Roots per transplant}

The data in below table (6) show that the Prosopis farcta, Folicist and Abo-najemh20 with their concentrations lead to increase the number of roots per transplant. Also, the high concentration had a significant difference in dual interactions between the studied factors. In case of triple combination, it clearly found that the 200 ppm Prosopis farcta +100 ppm Folicist + $150 \mathrm{ppm}$ Abo-najemh20 was superior combination treatment affecting the number of roots per pomegranate transplant which recorded (12\%) compared with control that was (3.67\%). 
Table (6): Effect of Prosopis farcta, Folicist and Abo najemh20 and their interactions on Root Number of Pomegranate transplant

\begin{tabular}{|c|c|c|c|c|c|c|}
\hline Prosopis farcta & Folicist & $\begin{array}{c}\text { Abo- } \\
\text { najemh20 } \\
0 \text { ppm }\end{array}$ & $\begin{array}{c}\text { Abo- } \\
\text { najemh20 } \\
75 \text { ppm }\end{array}$ & $\begin{array}{c}\text { Abo- } \\
\text { najemh20 } \\
150 \text { ppm } \\
\end{array}$ & $\begin{array}{c}\text { Prosopis } \\
\text { farcta * } \\
\text { Folicist }\end{array}$ & Prosopis farcta \\
\hline \multirow[t]{3}{*}{0} & 0 & $3.67 n$ & $5.67 \mathrm{kl}$ & $6.33 \mathrm{jk}$ & $5.22 \mathrm{f}$ & $5.85 \mathrm{c}$ \\
\hline & 50 & $4.33 \mathrm{mn}$ & $6.33 \mathrm{jk}$ & $7.33 \mathrm{~g}-\mathrm{j}$ & $6.00 \mathrm{e}$ & \\
\hline & 100 & $4.33 \mathrm{mn}$ & $6.33 \mathrm{jk}$ & $8.33 \mathrm{e}-\mathrm{g}$ & $6.33 \mathrm{e}$ & \\
\hline \multirow[t]{3}{*}{100} & 0 & $4.00 \mathrm{mn}$ & $5.00 \mathrm{Im}$ & $6.67 \mathrm{i}-\mathrm{k}$ & $5.22 \mathrm{f}$ & $6.63 \mathrm{~b}$ \\
\hline & 50 & $5.00 \mathrm{Im}$ & $7.67 \mathrm{f}-\mathrm{i}$ & $8.33 \mathrm{e}-\mathrm{g}$ & $7.00 \mathrm{~d}$ & \\
\hline & 100 & $6.67 \mathrm{i}-\mathrm{k}$ & $7.67 \mathrm{f}-\mathrm{i}$ & $8.67 \mathrm{~d}-\mathrm{f}$ & $7.67 \mathrm{c}$ & \\
\hline \multirow[t]{3}{*}{200} & 0 & $7.00 \mathrm{~h}-\mathrm{j}$ & $7.67 \mathrm{f}-\mathrm{i}$ & $8.67 \mathrm{~d}-\mathrm{f}$ & $7.78 \mathrm{c}$ & $9.30 \mathrm{a}$ \\
\hline & 50 & $8.00 \mathrm{e}-\mathrm{h}$ & $9.00 \mathrm{de}$ & $9.67 \mathrm{~cd}$ & $8.89 \mathrm{~b}$ & \\
\hline & 100 & $10.33 \mathrm{bc}$ & $11.33 \mathrm{ab}$ & $12.00 \mathrm{a}$ & $11.22 \mathrm{a}$ & \\
\hline \multicolumn{2}{|c|}{ Abo-najemh20 } & $5.93 \mathrm{c}$ & $7.41 \mathrm{~b}$ & $8.44 \mathrm{a}$ & & Folicist \\
\hline Prosopis farcta & 0 & $4.11 \mathrm{~h}$ & $6.11 \mathrm{f}$ & $7.33 \mathrm{de}$ & & \\
\hline \multirow[t]{2}{*}{${ }^{*}$ Abo-najemh20 } & 100 & $5.22 \mathrm{~g}$ & $6.78 \mathrm{e}$ & $7.89 \mathrm{~cd}$ & & \\
\hline & 200 & $8.44 \mathrm{c}$ & $9.33 b$ & $10.11 \mathrm{a}$ & & \\
\hline Folicist & 0 & $4.89 \mathrm{e}$ & $6.11 \mathrm{~d}$ & $7.22 \mathrm{c}$ & 0 & $6.07 c$ \\
\hline \multirow[t]{2}{*}{ * Abo-najemh20 } & 50 & $5.78 \mathrm{~d}$ & $7.67 \mathrm{c}$ & $8.44 \mathrm{~b}$ & 50 & $7.30 \mathrm{~b}$ \\
\hline & 100 & $7.11 \mathrm{c}$ & $8.44 b$ & $9.67 \mathrm{a}$ & 100 & $8.41 \mathrm{a}$ \\
\hline
\end{tabular}

Means of each factor and their interactions followed with the same letters are not significantly different from each other's according to Duncans multiple range test at 5\% level.

\subsection{Root length (mm)}

Table (7): Effect of Prosopis farcta, Folicist and Abo najemh20 and their interactions on Root length of Pomegranate transplant

\begin{tabular}{|c|c|c|c|c|c|c|}
\hline Prosopis farcta & Folicist & $\begin{array}{c}\text { Abo- } \\
\text { najemh20 } \\
0 \text { ppm }\end{array}$ & $\begin{array}{c}\text { Abo- } \\
\text { najemh20 } \\
75 \text { ppm }\end{array}$ & $\begin{array}{c}\text { Abo- } \\
\text { najemh20 } \\
150 \text { ppm }\end{array}$ & $\begin{array}{c}\text { Prosopis } \\
\text { farcta * } \\
\text { Folicist }\end{array}$ & Prosopis farcta \\
\hline \multirow[t]{3}{*}{0} & 0 & $11.00 p$ & 15.000 & $18.00 n$ & $14.67 \mathrm{f}$ & $20.48 \mathrm{c}$ \\
\hline & 50 & $21.00 \mathrm{Im}$ & $23.33 \mathrm{i}-1$ & $24.67 \mathrm{~h}-\mathrm{j}$ & $23.00 \mathrm{~d}$ & \\
\hline & 100 & $22.33 \mathrm{j}-\mathrm{m}$ & 22.00 k-m & 27.00 e-h & $23.78 \mathrm{~d}$ & \\
\hline \multirow[t]{3}{*}{100} & 0 & $12.00 p$ & $17.33 n$ & $20.67 \mathrm{~m}$ & $16.67 \mathrm{e}$ & $22.85 \mathrm{~b}$ \\
\hline & 50 & $23.67 \mathrm{i}-\mathrm{k}$ & $25.33 \mathrm{~g}-\mathrm{i}$ & $27.33 \mathrm{e}-\mathrm{g}$ & $25.44 \mathrm{c}$ & \\
\hline & 100 & $24.67 \mathrm{~h}-\mathrm{j}$ & $26.33 \mathrm{f}-\mathrm{h}$ & 28.33 ef & $26.44 \mathrm{c}$ & \\
\hline \multirow[t]{3}{*}{200} & 0 & $12.00 p$ & $26.67 \mathrm{f}-\mathrm{h}$ & 28.67 ef & $22.44 \mathrm{~d}$ & $30.04 \mathrm{a}$ \\
\hline & 50 & $29.33 \mathrm{e}$ & $31.67 \mathrm{~d}$ & $33.33 \mathrm{~cd}$ & $31.44 \mathrm{~b}$ & \\
\hline & 100 & $34.67 \mathrm{bc}$ & $36.33 \mathrm{ab}$ & $37.67 \mathrm{a}$ & $36.22 \mathrm{a}$ & \\
\hline \multicolumn{2}{|c|}{ Abo-najemh20 } & $21.19 \mathrm{c}$ & $24.89 \mathrm{~b}$ & $27.30 \mathrm{a}$ & & Folicist \\
\hline Prosopis farcta & 0 & $18.11 \mathrm{f}$ & $20.11 \mathrm{e}$ & $23.22 \mathrm{~d}$ & & \\
\hline \multirow[t]{2}{*}{${ }^{*}$ Abo-najemh20 } & 100 & $20.11 \mathrm{e}$ & $23.00 \mathrm{~d}$ & $25.44 \mathrm{c}$ & & \\
\hline & 200 & $25.33 c$ & $31.56 \mathrm{~b}$ & $33.22 \mathrm{a}$ & & \\
\hline \multirow{3}{*}{$\begin{array}{c}\text { Folicist } \\
\text { *Abo-najemh20 }\end{array}$} & 0 & $11.67 \mathrm{~g}$ & $19.67 \mathrm{f}$ & $22.44 \mathrm{e}$ & 0 & $17.93 \mathrm{c}$ \\
\hline & 50 & $24.67 d$ & $26.78 \mathrm{c}$ & $28.44 \mathrm{~b}$ & 50 & $26.63 \mathrm{~b}$ \\
\hline & 100 & $27.22 b c$ & $28.22 \mathrm{~b}$ & $31.00 \mathrm{a}$ & 100 & $28.81 \mathrm{a}$ \\
\hline
\end{tabular}

Means of each factor and their interactions followed with the same letters are not significantly different from each other's according to Duncans multiple range test at 5\% level.

The length of pomegranate transplant roots was strongly affected by spraying of Prosopis farcta, Folicist and Abo-Najemh20. The dual and triple interactions also play a significant role in improving the length of roots especially at third levels (high concentrations of studied factors) 


\section{DISCUSSION}

It is clear from data that in above tables, the effect of Folicist on growth of pomegranate transplant characteristics was positive effect in all studied parameters, the results may be due to the role of essential elements in transplants such as photosynthesis reactions, DNA metabolism, and protein and carbohydrate biosynthesis due to increased mineral content in leaves (Hafez \& ElMetwally, 2007). Furthermore, folicist Enhances $\mathrm{N}$ uptake as well as improving photosynthesis in fruit trees. Nitrogen is known to be an essential component of many compounds in plants such as chlorophyll, nucleotides, proteins, alkaloids, enzymes, hormones, and vitamins. (Marschner, 2011; Saeed \&Mayi 2016)

\section{CONCLUSIONS}

Based on the results in this study it found that the Prosopis farcta at $200 \mathrm{ppm}$ had a significant difference compared with control affecting on all studied parameters. The spraying of Folicist and Abo-Najemh20 with their concentration also make high enhancement in most of studied parameters. The best dual interaction affecting studied parameters founded when using high concentration of three factors. Then, the interaction among $200 \mathrm{ppm}$ of Prosopis farcta, $100 \mathrm{ppm}$ of Folicist and $150 \mathrm{ppm}$ of Abo-najem h 20 had a significant effect of all studied parameters.

So according to the obtained results it highly recommended to use the Prosopis farcta with other fruit trees or plants due to its positive effect on growth and it don't lead to contaminate the environment.

\section{REFERENCES}

Al-Rawi, K. M., \& Khalafalla, A. (2000). Analysis of Experimental Agriculture Design. Dar AlKutub for Printing and Publishing. Mosul Univ. Iraq (in Arabic). pp, 488.

Chaudhari, S.M. and Desai, U.T. 1993. Effect of plant growth regulators on flower sex in pomegranate. Ind. J. Agril. Sci., 63:34-35
Eissa Fawzia, M., Faith, M. A., \& El-Shall, S. A. (2007). The Role of humic acid and rootstock in enhancing salt tolerance of"Le-Conte"pear seedlings. J. Agric. Sci. Mansoura Univ, 32(5), 3651-3666.

Ghosh, S. N., Bera, B., Roy, S., \& Kundu, A. (2009). Effect of plant growth regulators in yield and fruit quality in pomegranate cv. Ruby. Journal of Horticultural Sciences, 4(2), 158-160.

Hafez, O. M., \& El-Metwally, I. M. (2007). Efficiency of zinc and potassium sprays alone or in combination with some weed control treatments on weeds growth, yield and fruit quality of Washington navel orange orchards. J. Appl. Sci. Res, 3(7), 613-621.

Harzallah-Skhiri, F., \& BenJannet, H. (2005). Flavonoids diversification in organs of two prosopis farcta (banks \& sol.) eig.(leguminosea, mimosoideae) populations occurring in the northeast and the southeast of Tunisia. J Appl Sci Res, 1(2), 130-36.

Marschner, H. (2011). Marschner's mineral nutrition of higher plants. Academic press.

Pasiecznik, N. M., Harris, P. J., \& Smith, S. J. (2004). Identifying tropical Prosopis species: a field guide (p. 29). Coventry: HDRA Publishing.

PASIECZNIK, N.M. 2001. Prosopis - management by exploitation, not eradication, required to control weedy invasions. ACOTANC 2001, the 9th Australian Conference on Tree and Nut Crops, 13-19 April 2001, Perth, Australia (www.AOI.com.au/acotanc/papers).

Saeed, G. M., \& Mayi, A. A. (2016). Effect of Pro. Sol, Folicist and Cultivar on Leaf Nutrients of Olive (Olea europaeaL.) Transplants.

SAS Institute. (2015). Base SAS 9.4 procedures guide. SAS Institute.

Sharma, J., Ramchandra, K. K., Sharma, D., Meshram, D. T., \& Ashis Maity, N. N. (2014). Pomegranate Cultivation Marketing and Utilization.

Sharma, J., Ramchandra, K. K., Sharma, D., Meshram, D. T., \& Ashis Maity, N. N. (2014). Pomegranate Cultivation Marketing and Utilization.

Ziosi, V., Zandoli, R., Vitali, F., \& Nardo, A. D. (2013). FOLICIST®, a biostimulant based on acetyl-thioproline, folic acid and plant extracts, improves seed germination and radicle extension. Acta Horticulturae, (1009), 79-82. 
كارتيّكرنا رهشاندنا دهرخستيى رووهكي خورنبفكي وفوليسيت وئهبونهجم لسهر گه شهيا نهمامكيّن هناريّ جوري ووندرفول

يوخته

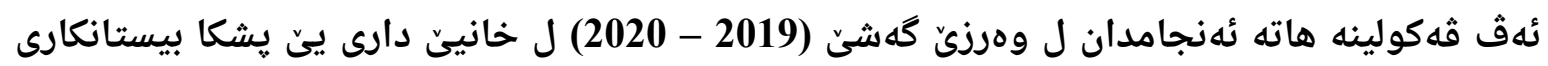

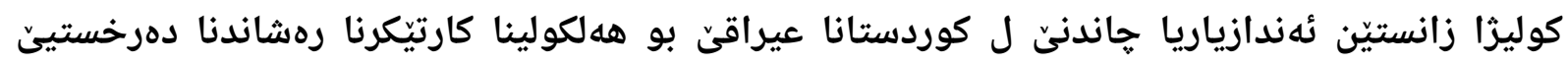

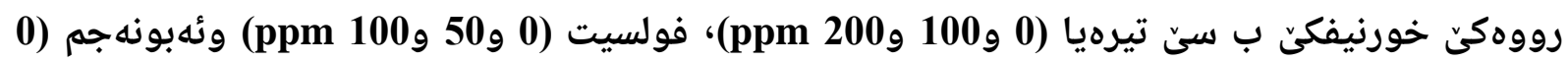
و75 و150 لسهر گهشهيا نهمامكيّت هناريّ. ئهنجاما دياركر كو ههمى ساخلهتيّن هاتينه وهركرتن

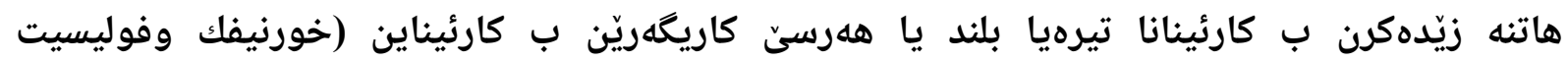

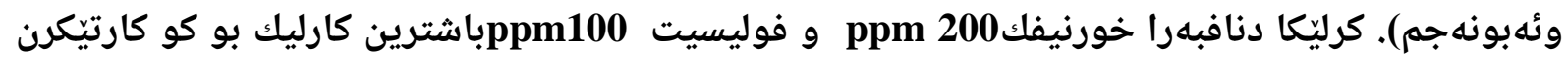
لسهر ساخله تان كرى وكارليّك دنافبهرا

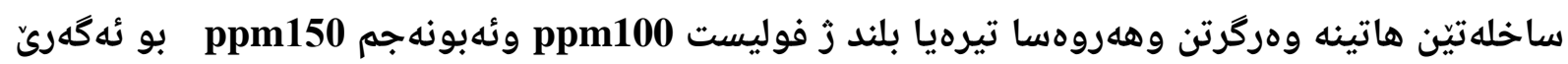

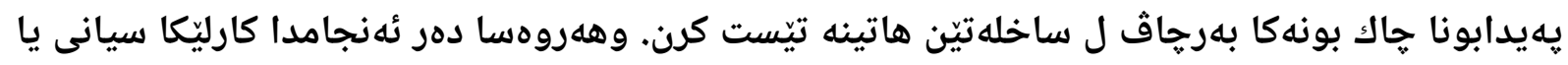

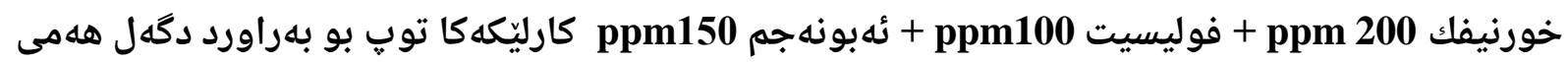
كارليّكيّن دى وهلروهسا دگهل كارليّكا كونترولى زئ.

تأثير الرش مستخلص نبات الخرنوب و فوليسيت و أبونجم اتش 20 على نمو شتلات الرمان صنف وندرفول

الخلاصة

أجريت هذه الدراسة في موسم النمو 2019 - 2020 في ظلة الخشبية تابعة ل قسم البستنة، كلية علوم

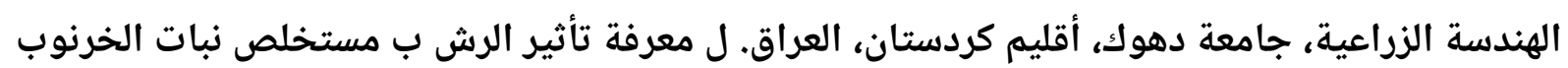
بتراكيز (0 100200 جزء من المليون) وفولسيت بتراكيز (150 50100 جزء من المليون) وأبونجم 20 بتراكيز (الم 75150 جزء من المليون) على شتلات الرمان صنف وندرفول. أظهرت النتائج بانه جميع الصفات المدروسة تحسنت تحسنا معنويا ب تراكيز العالية من العوامل المأخوذة وفي التداخل الثنائي والثلاني الثلاثي أيضا هنالك تحسين في الصفات المدروسة ب استعمال التراكيز العالية من الخرنوب وفوليسيت وأبو نجم 